brought into the vicinity of the part involved, and with the orderly supporting the screen the rays are turned on and the lights off. The foreign body is now accurately "centred," and to effect this a small diaphragm is employed. The movements of the tube box can be accomplished with the operator's foot, and these are rendered easier if a toecap to receive the point of the boot be fixed to the front of the box. The point of a pair of Spencer Wells forceps is now laid on the skin so that it coincides with the shadow on the screen. The light is now turned on and the screen removed. With due regard to anatomical considerations the incision is made, not necessarily immediately over the foreign body, for, indeed, an oblique approach is often easier. The skin incision should be fairly free, and when the muscles have been exposed blunt dissection can be employed. No violence in the extraction is permissible, and should the foreign body be in the vicinity of important structures free and careful dissection in a good light is demanded, the operation being merely controlled by occasional reference to the screen. The forceps employed here for actually extracting foreign bodies are usually Kocher's or long Spencer Wells's. As a guide to the correct estimation of the depth of the foreign body it is extremely useful, while the forceps are approaching to move the tube box from side to side with the foot. The relative movement of the foreign body and the point of the forceps indicates their distance apart; for example, if the ball apparently moves somewhat faster than the point of the forceps, the ball lies somewhat deeper. With experience it is scarcely ever necessary to move the tube, more particularly in the case of the extremities, for here a slight rotatory movement of the part supplies the necessary information. The extraction having been performed, the wound is sutured in the ordinary way.

In septic cases, where a sinus exists, the preliminary cleansing of the part and of the sinus is of the utmost importance. For this purpose we employ a triple irrigating apparatus; three irrigators mounted on a stand are connected so that each in turn may be made to discharge viâ one cannula.

The solutions employed are: (1) Biniodide of mercury ( 1 in 1000); (2) carbolic acid ( 1 in 40); and (3) hydrogen peroxide ( 1 in 3 ). The wound is copiously irrigated with these one after the other. This treatment may seem drastic, but is no more than is merited by the frightful septicity with which we so frequently have to deal. The actual extraction of the foreign body is now performed, but here free incisions are very desirable. It is usual to approach the foreign body by an incision which will, by reason of a dependent situation, afford the best drainage possible. In more quiescent cases an approach via the sinus is frequently sufficient. It is often of value to leave the forceps and the foreign body in situ, and enlarge the tract freely before extracting; in this way foreign matters, such as cloth, \&c., perhaps of greater importance even than shrapnel and bullet, can be in many cases removed.

As indicating the general applicability of the method, we may mention that with the guidance of the rays we have removed foreign bodies from the posterior mediastinum and from the sphenomaxillary fossa; then there was a pelvic case in which two previous abortive efforts had been made to extract a bullet. In this case we successfully removed the bullet interior from the region of the great sacro-sciatic ligament and the mantle of the same bullet from behind the neck of the femur. Foreign bodies in the neighbourhood of the spine and in medulla of bones have like. wise been removed with a success which is all the more gratifying as many of these cases showed evidence of previous attempts. In multiple shell wounds this method of treatment gives much satisfaction; in these cases each wound in itself seems trifling, but the sum total of upwards of six suppurating foci induces such a toxæmic state that prolonged anæsthesia and much interference are very undesirable. We prefer also to operate on compound fractures on the $X$ ray table, as the steps of the operation from start to finish can be controlled and guided by reference to the screen. The value of the method is unquestionable, and while we cannot claim for it originality, we are not aware that it has become a routine method of treatment in any other hospital. With a little experience it becomes possible to remove minute fragments which in the ordinary way would necessitate frightful mutilation.

In conclusion, 1 wish to acknowledge my in. debtedness to Mr. C. M. Hewer, surgeon to the hospital, for his invaluable aid in meeting the many difficulties which arose in the evolution of the $\mathrm{X}$ ray theatre.

\section{A RE-INVESTIGATION ON THE NATURE OF THE CELLULAR ELEMENTS} PRESENT IN MILK.*

\author{
BY R. TANNER HEWLETT, M.D. LOND., \\ F.R.C.P. LOND., D.P.H.,
}

PROFESSOR OF BACTERIOLOGY IN THE UNIVERSITY OF LONDON; AND

CECIL REVIS, A.C.G.I.,

CHIEF CHEMTST TO MESSRS. WELFORD AND SONS, LIMTIED, LONDON.

IN a series of papers Hewlett, Villar, and Revis ${ }^{1}$ recorded the results of investigations on the nature of the cellular elements present in milk. It was shown that cells of various kinds are always present in the milk of perfectly healthy cows, sometimes in enormous numbers, and that no reason could be assigned for the considerable variation in the number of the cells which occurs in the milk of different cows. A preliminary classification of these cells was formulated, and the opinion was expressed that the vast majority of them are not leucocytes, and that the "multinucleated cells" which are frequently present in considerable numbers and have a superficial resemblance to poly. morphonuclear leucocytes are for the most part the "germinal cells" of Winkler derived from the epithelial layer of the glandular tissue of the udder and are embryonic cells which develop into epithelial cells.

Breed $^{2}$ has recently again stated that these cells are polymorphonuclear leucocytes derived from the blood, and asserts that the evidence formerly adduced by Hewlett, Villar, and Revis is not convincing. As, however, Breed produces no evidence at all in support of his statement, and also having regard to the importance of the subject from the public health point of view, we have considered it

* This investigation has bee carried out in the Bacteriological Laboratories of King's College, London, and of Messrs. Welford and Sons, with the aid of a grant from the Medical Research Committee. 1 Journal of Hygiene, ix., p. 271 ; x., p. 56 ; xi., p. 97 ; xili., p. 87 (Bibliography)

2 New York Experiment Station, Bulletin 380, 1914. 
advisable to re-investigate the nature of the cellular elements present in milk, to revise their classifica. tion, and to attempt to determine the factors which influence their number in the milk of different cows.

With the object of elucidating the nature of the cells and to revise their classification a large number of trials of various methods of preparing, fixing, and staining films has been made, and it is proposed first to detail the results of this part of the investigation.

\section{Obtaining the Cells.}

The cells must be obtained by centrifuging the milk, but it is found that the number of cells thus obtained falls far short of the actual number which deposit after a preliminary treatment of the milk. This preliminary treatment may consist either in warming the milk to $60^{\circ}-70^{\circ} \mathrm{C}$. for 10 minutes or by the addition of a small quantity $(0.5$ per cent.) of formalin to the milk and allowing the mixture to stand for 24 hours before rotating. The latter was the method adopted in our previous investigations, but the question arises whether some alteration of the cells is not produced thereby. We have made comparative examinations of the cells obtained from milk with and without the addition of formalin, and there is no doubt that even the small quantity of formalin formerly employed does sometimes cause decided contraction of the cells. We have therefore in the following investigation discarded the use of formalin. We find also that a considerable proportion of the cells, and in particular that variety of them which we have provisionally named the "multinucleated," are carried up in the cream-layer, and if the cream-layer be broken up and the milk rotated again, a further yield of cells is obtained; but the process must be repeated three or four times (the breaking up of the cream-layer and subsequent rotation) if even a majority of the cells is to be obtained in the deposit.

\section{Preparation of the Films.}

After the deposit of cells has been obtained by centrifuging, they need to be washed so as, so far as possible, to remove the milk and fat admixed with them. The supernatant milk is decanted from the deposit of cells and the fat wiped out of the tube. The cells are then washed. We naturally at first employed physiological salt solution for this purpose, but a study of the results so obtained undoubtedly points to the conclusion that some alteration and distortion of the cells occurs when the film is made and allowed to dry, probably from the concentration of the salt which must inevitably take place as the water evaporates.

As the shape of the cells will be materially affected by the tonicity of the fluid in which they are placed, we were at first inclined to believe that the osmotic pressure of ordinary physiological salt solution was different from that of the milk in which the cells at first find themselves. From the freezing point of milk it may be deduced that its osmotic pressure corresponds to that of a saline solution of 0.9 per cent. concentration. Experiments were therefore made with solutions of sodium chloride of $0.7,0.8,0.9$, and 1.0 per cent. concentrations, but there appeared to be no appreciable difference in the resulting film. The same distortion or alteration was still noticeable. It was then established that the salt solution in itself had no such influence, as, if the deposit after washing with salt solution was then washed with ox serum (see below) the cells were normal in aspect. It seemed, therefore, that the drying in films was really responsible for the alteration, though it is noteworthy that the tendency is rather towards an expansion of the cell than a contraction, as would be expected in a solution which was rapidly concentrating.

Another modification employed for washing was an alkaline physiological salt solution. Sodium carbonate $\left(\mathrm{Na}_{2} \mathrm{CO}_{3}\right)$ was used for this purpose, so that the final alkalinity corresponded to $\mathrm{N} / 35 \mathrm{Na}_{2} \mathrm{CO}_{8}$ (which is approximately that which obtains in blood serum, according to Wright) and the Na-ion concentration was made approximately equal to $0^{\circ} 9$ per cent. NaCl. Other modifications of the wash-liquid were (a) solution of egg albumin adjusted by the addition of distilled water to 7.25 per cent. of protein, so as to correspond with the protein content of ox serum; (b) egg albumin and sodium chloride, so as to contain 7.25 per cent. of protein and a Na-ion concentration equal to that of 0.9 per cent. solution of sodium chloride; and (c) ox serum, both unheated and heated to $56^{\circ} \mathrm{C}$. for half an hour. None of these presents any special advantage with the exception of ox serum. We find that washing with heated ox serum gives undoubtedly the best results, causing the least contraction of the cells of any washing agent employed. The aspect of the cells obtained from the same milk, washed in the one case with saline and in the other with ox serum, is entirely different, and the cells would scarcely be recognised as of similar origin. The effect of saline is most marked on nuclear structures.

\section{Preparation of the Films.}

The cellular deposit after washing and centrifuging is deposited on a clean slide and spread with a glass rod so as to form a thin even film. This is allowed to dry in the air or by warming to $37^{\circ} \mathrm{C}$, and the slide with the dry film upon it is then immersed in a mixture of equal parts of alcohol and ether, to remove the fat, for from half an hour to several hours; it is then ready for staining.

\section{Staining the Film.}

A number of different staining methods has been tried, the stains employed being Jenner's stain, Leishman's stain, Pappenheim's stain, Giemsa stain (Revis's method ${ }^{3}$ ), Giemsa stain (Coles's method ${ }^{4}$ ), Ehrlich's tri-acid stain, hæmatoxylin and eosin, and the May-Grünwald stain. Of all these, the May-Grünwald stain ${ }^{5}$ undoubtedly gives the best results. This is carried out as follows: One tablet of the May-Grünwald stain is dissolved in 100 c.c. of pure methyl alcohol (this solution keeps well). of this solution, 0.3 c.c. is dropped on the film and allowed to act for one to two minutes, then 0.9 c.c. of specially distilled water is dropped on and is allowed to act for three to six minutes. The film is finally washed in distilled water and dried in the blood-heat incubator. In order to prevent the stain running all over the slide it is useful to make a streak at either end of the film with a soft paraffin mixture composed of vaseline 75 parts and paraffin wax 25 parts, and melted in hot water for use.

We are now trying the method described by Scott for blood films, ${ }^{6}$ in which the films are not allowed

3 Journal of Hvgiene 1910, vol.x., p 83.

4 See Hewlett's Manual of Bacteriology, fifth edition, 1914, p. 525. 5 The May-Grinwald stain is made as fullows: $100 \mathrm{ccc}$. of a 1 per cent. solution of eosin are mixed with 100 c.c. of a solution of methslene blue and allowed to stand 24 hours. The precipitate is washed with water and dried and made up into tablets of 0.25 gramme each.

Journal of Pathology and'Bacteriology, 1901, vol. vii.; p. 233. 
to dry but are fixed with formalin vapour and the staining is carried out on the wet film, and anticipate that it may give still better results. Undoubtedly the nuclear structure revealed by this method is very different and much more complex than that brought out by the other methods.

A point of importance, and one which perhaps has not received sufficient attention, is that the aspect of the cells differs considerably according to the staining method employed. This applies not only to the cells of milk, but also to the leucocytes of ox blood (which we are also studying) which we have employed to control our methods. The actual tint of the various structures is also markedly affected by the reaction of the fluid used to wash the deposit in, so that films of cells washed in saline showed very different results from those washed in ox serum. It is quite certain that some method of at once hardening the cell wall in order to obviate distortion and disruption is essential to the proper study of these cells. It is not improbable that the cells as they appear in ordinary films are quite different in appearance from that which they really possess. For this reason the method of Scott, though somewhat more difficult of application than ordinary methods, has manifest advantages. The statement commonly made that the cells of milk stain well and naturally with ordinary blood stains is, in our experience, quite erroneous.

\section{Influence of "Keeping" on the Cells.}

The cells present in milk may obviously either be "recent" or may have gained access to the milk from the period immediately succeeding the last previous milking. The question arises, Will such a sojourn in the milk alter these cells? It is difficult definitely to answer this question. We know that leucocytes may be kept in physiological salt solution apparently unaltered for many hours, and we find that ox leucocytes added to milk are apparently unaltered after a sojourn of 24 hours at room temperature. Similarly, the cells of milk examined immediately after milking and again examined after the same milk has been kept at room temperature for 24 hours show little or no difference. The probability is therefore that the majority of the cells present in milk undergo little or no alteration during the period the milk remains in the udder.

\section{URINARY CALCULUS IN THE PELVIC PORTION OF THE URETER.}

BY JOSEPH E. ADAMS, M.S. LoND., F.R.C.S. ENG., SURGEON IN CHARGE OF OUT-PATIENTS, ST. THOMAS'S HOSPITAL, ETC.

CASES of calculus impacted in the ureter are of interest from the diagnostic point of view, especially when the stone lies in the right ureter, for in whichever portion it is situated the resemblance of the symptoms to those of appendicitis may easily lead to error. In the present paper I propose to call attention to some points in the differential diagnosis of calculi in the pelvic portion of the ureter and to describe what I consider to be the best route for the removal of such impacted stones.

The three common situations in which calculi become arrested in their descent from kidney to bladder is: (1) At the junction of the renal pelvis and ureter; (2) in the abdominal portion of the ureter, including that part which crosses the brim of the true pelvis; and (3) in the pelvic portion of the ureter. The second is the least common, the first can hardly be distinguished from calculus in the kidney itself, while the third gives rise to symptoms of a most variable nature. It is well known that calculi may remain impacted in the ureter for a considerable time, giving rise to no symptoms when once they have actually come to rest unless the intermittent obstruction which they cause leads to either hydro- or pyo-nephrosis. On the other hand, even stationary calculi may give rise to attacks of pain closely resembling typical renal colic asso. ciated with hæmaturia.

\section{Diagnosis.}

Most surgeons hare knowledge of cases where appendicectomy has been followed by the passage of a urinary calculus per urethram, and of instances of obscure abdominal pain where a similar natural relief of all symptoms has occurred. Such events, whilst satisfactory to the patient, should lead to humility in the surgeon and a determination not to neglect the aid of skiagraphy in doubtful cases. With regard to calculi impacted in the last few inches of the ureter. Thomson Walker ${ }^{1}$ states that symptoms of bladder irritation are prominent features, and that there is frequency of micturition with pain along the urethra to the end of the penis; also that genital symptoms may exist, such as painful emissions, bæmospermia, and testicular pain. The patient may complain of pain in the rectum, aggravated by defæcation. Undoubtedly the most constant symptom is pain; abdominal pain, pelvic pain, renal or ureteral colic may be associated with a calculus which has come to rest even in the pelvic portion of the ureter, and the most usual history is of severe attacks of pain in the lumbar region in the past and dull gnawing pain in the lower abdomen in the present history of illness. It is not uncommon for a fixed pelvic calculus to canse pain starting in the loin and extending to the hypogastrium. If definite attacks of pain occur they are usually accompanied by vomiting, sweating, and hæmaturia, but the last symptom is the least constant and may be observed only once or twice even where the attacks are numerous. Rest in bed frequently fails to give immunity against these attacks, as it does in ordinary renal colic. Some fever is apt to be present. As regards urinary symptoms, frequency is the most common, and a careful examination shortly after any acute attack of pain will usually reveal the presence of a trace of albumin and possibly some pus cells; hæmaturia, as already mentioned, occurs in some acute attacks. Tenderness and rigidity on palpation are not uncommon both in the lumbar region and the hypogastrium, even when the stone is impacted close to the bladder. The rigidity, of course, is rarely of the same degree as that found in peritoneal lesions. Hydronephrotic symptoms are comparatively rare, but if they are present they dominate the clinical picture, and before operation the actual cause of such hydronephrosis may not be suspected. The ultimate diagnosis usually rests with the result of $X$ ray examination although it may be possible in children to palpate a calculus in the pelvic portion of the ureter by rectal examination, or by the vagina in the female. When the stone lies impacted in the ureter behind the bladder wall cystoscopic examination is usually negative as regards the appearance of the ureteric orifices, but the passage of a ureteric bougie may be arrested, and this observation combined with skiagraphy is 